\title{
Bienestar psicológico en habitantes de calle resocializados de la ciudad de Medellín*
}

Psychological well-being in resocialized street residents of Medellín city

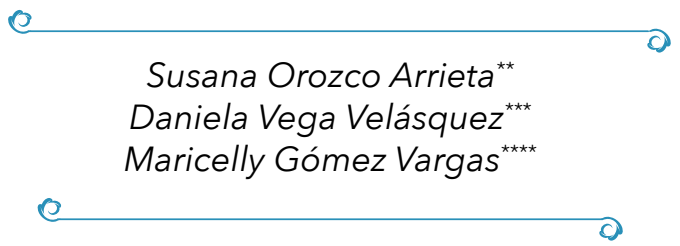

Recibido 12. 04. 2019• Arbitrado 25. 05. $2019 \bullet$

Aprobado 13.06. 2019

El artículo presenta los resultados del trabajo de grado de las estudiantes para optar el título de psicólogas

** Estudiante de psicología Universidad de Antioquia, susana.orozco@udea.edu.co

*** Estudiante de psicología Universidad de Antioquia, daniela.vega@udea.edu.co

**** Psicóloga, Magíster en Psicología, docente Universidad de Antioquia, Grupo de investigación Psyconex, Departamento de Psicología, Facultad de Ciencias Sociales y Humanas, Universidad de Antioquia, ORCID: 0000-0002-2389-7821, gomez@udea.edu.co

\section{Resumen}

El artículo presenta resultados de un estudio orientado a describir el bienestar psicológico en una muestra de habitantes de calle resocializados de la ciudad de Medellín, Colombia. El estudio de un enfoque cualitativo empleó una entrevista semiestructurada diseñada según el concepto teórico de Bienestar Psicológico, propuesto por Casullo (2002), compuesto por cinco dimensiones: aceptación de sí mismo, autonomía, control de situaciones, vínculos psicosociales y proyectos. Como estrategia metodológica se utilizó el estudio de caso, que permitió una aproximación a las dimensiones del bienestar psicológico en cuatro participantes mayores de edad de Medellín. Entre los resultados se destaca que en cada dimensión los participantes presentan elementos de cambio respecto a su anterior condición como habitante de calle; sin embargo, todavía hay aspectos relacionados con la capacidad para tomar decisiones y de autorregulación que ameritan un acompañamiento profesional, vinculando a la familia y red de apoyo, y manteniendo como eje transversal la ayuda al prójimo, sentido de vida fundamental que coincidió para todos los entrevistados.

Palabras clave: Habitante de calle, bienestar psicológico, resocialización 


\section{Abstract}

The article presents the results of a study aimed at describing psychological well-being in a sample of resocialized street residents of the city of Medellin, Colombia. The study of a qualitative approach used a semi-structured interview designed according to the theoretical concept of Psychological Wellbeing, proposed by Casullo (2002), composed of five dimensions: acceptance of self, autonomy, control of situations, psychosocial links and projects. As a methodological strategy, the case study was used, which allowed an approximation to the dimensions of psychological well-being in four participants older than Medellin. Among the results it is highlighted that in each dimension the participants present elements of change with respect to their previous condition as street dwellers; However, there are still aspects related to the ability to make decisions and self-regulation that merit professional accompaniment, linking the family and support network, and maintaining as a transversal axis the help of others, a fundamental sense of life that coincided for all interviewed.

Keywords: Street dweller, psychological well-being, resocialization

\section{Introducción}

Los habitantes de calle conforman una población que encuentra en el espacio público un lugar en el cual desarrollar su vida. Dicha población se caracteriza por ser constantemente marginada y expuesta a las consecuencias de la exclusión social, siendo el rechazo, el temor y la indiferencia las principales reacciones de otros ciudadanos hacia ellos. Por otro lado, la situación de habitar la calle tiene sus raíces en la estructura económica imperante en los países de América Latina y se incrementa debido a factores políticos y sociales. En el caso específico de Colombia, el aumento constante de esta problemática está íntimamente relacionado con la desarticulación social, que trae consigo desigualdad e inequidad con sus correspondientes secuelas: violencia y pobreza (Correa, 2007).

Teniendo en cuenta lo anterior, se ha tratado de determinar la cantidad de habitantes de calle existentes en todo el territorio nacional y esto ha permitido evidenciar una mayor concentración de esta población en las principales ciudades del país, como lo son Bogotá, Cali y Medellín; identificando para esta última unas cifras mayores en relación con los otros dos lugares. Para el año 2002, en Medellín había 3.483 habitantes de calle (DANE, 2002) y aunque en el año 2009 esta cantidad disminuye a 3.381 (Secretaria de Bienestar Social y CEO UdeA, 2009), ella no es muy significativa, representando esto una 
preocupación para las administraciones gubernamentales que trabajan con miras a la rehabilitación social de las personas que habitan la calle y que, pese a ello no lo logran eficacia. Cabe añadir que a la fecha no hay datos actualizados en cuanto a este grupo poblacional, ni en Medellín, ni a nivel nacional.

Por su parte, la revisión bibliográfica de antecedentes llevada a cabo en el marco del estudio realizado permite apreciar claramente una tendencia centrada en la identificación de las características sociodemográficas de los habitantes de calle (Arias y Pamplona, 2015; Barreat, 2007; Agudelo y Sánchez, 2012). En cuanto a las temáticas investigativas que predominan respecto a la población habitante de calle, se encontraron asuntos como la multicausalidad de la vida en calle y la manera en que el habitante de calle significa diversos asuntos de la vida (González, Blandón, Quiceno, Giraldo y Forero, 2014); la diversidad en las motivaciones para ingresar a un componente de resocialización (Agudelo y Sánchez, 2012) y las problemáticas presentes en las prácticas de intervención realizadas para habitantes en situación de calle (Di Lorio, Seidman, Gueglio y Rigueiral, 2016), y sólo se encontró un estudio sobre personas resocializadas en Medellín, indagando sobre los factores que les permitía mantenerse en esa condición y no retornar a la calle (Calderón, Gómez, Dávila y Zapata, 2018).

De este modo, los antecedentes revisados no solo permitieron un acercamiento al recorrido investigativo en torno a la población habitante de calle, sino que, además, indicaron que, si bien hay un interés acerca de asuntos diversos concernientes a esta población, se ha dejado de lado la referencia a los aspectos positivos de la persona, mismos que pueden ser determinantes en el logro o fracaso de un proceso de resocialización. Es por esta razón que la investigación se centró en destacar las dimensiones del Bienestar Psicológico en personas que fueron habitantes de calle y que son reconocidos como resocializados, término criticado pues connota un sujeto por fuera de la sociedad, y ante lo cual las autoras no concuerdan, pero que debe ser usado así, pues se trata de un término institucionalizado que coincide con la última fase del proceso de atención del Sistema de Habitante de Calle en Medellín (Restrepo y Storti, 2011).

El concepto de Bienestar Psicológico, en adelante BP, tuvo un recorrido histórico iniciado con los debates académicos de la tradición eudaimónica aristotélica (Díaz et al., 2006; Vielma y Alonso, 2010). Así mismo, han sido diversos los autores que han contribuido a la construcción de conocimiento acerca del BP, siendo la psicóloga Carol Ryff una de sus principales exponentes. A pesar de que las teorizaciones del concepto son variadas y acuñan diversos 
términos, la mayoría de las definiciones coinciden en que está orientado hacia el desarrollo del potencial humano y el crecimiento personal, los cuales están estrechamente relacionados con la realización de metas, el establecimiento de relaciones interpersonales basadas en la confianza y la consciencia de un propósito de vida, además de ser un indicador positivo de salud mental. Cabe mencionar que la investigación se fundamentó en la teorización de Casullo (2002) sobre BP definiéndolo como "la percepción que una persona tiene sobre los logros alcanzados en su vida, del grado de satisfacción personal con lo que hizo, hace o puede hacer, desde una mirada estrictamente personal" (p. 11). Además, propone que el estudio del BP debe contemplar el análisis de aspectos referentes a la aceptación de sí mismo, la autonomía, el control de situaciones o dominio del entorno, el establecimiento de vínculos psicosociales y la capacidad de plantearse proyectos. Dichos elementos se asemejan a las dimensiones ya propuestas por Ryff, excepto una sexta dimensión denominada crecimiento personal propuesta por Casullo. Fue privilegiada la teorización de Casullo, porque sus investigaciones de BP en ciertos grupos poblacionales en Iberoamérica se asumieron como los principales referentes para el análisis y discusión de los resultados del presente estudio (Castro y Casullo, 2001; Casullo y Castro, 2000).

Con base en la evidente problemática que representa el fenómeno de habitar la calle y en la marcada tendencia investigativa que poco se centra en aspectos concernientes a la salud mental en esta población y sus condiciones subjetivas, se llevó a cabo una investigación para identificar la presencia de aspectos relativos al BP en personas que fueron habitantes de calle de la ciudad de Medellín y que lograron su resocialización. Dicho objetivo pretendió no sólo aportar a la comprensión del fenómeno desde otra perspectiva, sino que también, de manera más específica, se acercó a asuntos que las personas entrevistadas definieron como constituyentes de su BP, particularizando aquellos elementos invisibilizados en esta población y que podrían ser fortalecidos y potencializados en quienes aún se encuentran en condición de habitante de calle, para lograr su resocialización.

Finalmente, el conocimiento de los asuntos que generan bienestar en las personas resocializadas es un insumo valioso para las instituciones cuya labor está enfocada en la atención al habitante de calle, por lo cual se espera que los hallazgos de la investigación contribuyan al fortalecimiento del trabajo que se viene realizando desde los ejes de prevención, mitigación y superación del fenómeno. Todo lo anterior en su conjunto, representa los aportes que la investigación plantea tanto a nivel teórico como práctico. 


\section{Método}

\section{Diseño}

El fenómeno se abordó desde un enfoque cualitativo-hermenéutico, valiéndose del estudio de caso como estrategia investigativa (Galeano y Vélez, 2002), la cual permitió una aproximación del Bienestar Psicológico en personas que fueron habitantes de calle de la ciudad de Medellín y que actualmente se encuentran resocializados.

\section{Participantes}

La muestra para el estudio fue seleccionada de manera intencional, constituyéndose por cuatro personas: dos de ellos de sexo masculino de 50 y 59 años y dos de sexo femenino con edades de 38 y 56 años. Como criterios de inclusión, se consideró que los participantes fueran mayores de edad, que no padecieran de un trastorno mental severo y que tuvieran como mínimo 6 meses de condición como resocializado, es decir, que habían superado el último paso en el proceso llevado a cabo al interior del Sistema Habitante de Calle. Y finalmente, que quisieran participar de manera voluntaria en la investigación.

\section{Instrumentos}

Se diseñó una entrevista semiestructurada sustentada en la teoría del Bienestar Psicológico y las cinco dimensiones planteadas por Casullo (2002) acorde a los objetivos contemplados en el proyecto. El instrumento fue evaluado por dos expertos, ambos profesionales de la Psicología con experiencia en el trabajo con habitante de calle.

\section{Procedimiento}

Se contactaron a los participantes por vía telefónica y luego de firmar los consentimientos informados se solicitó la grabación de cada entrevista, la cual duró en promedio una hora. Luego se transcribieron y se sistematizaron en Word, se realizó la codificación en Excel y posteriormente se analizaron los datos a partir de las categorías analíticas y emergentes en su discusión permanente con los datos primarios, la teoría de Casullo (2002) y la revisión de otras referencias bibliográficas sobre el tema. 


\section{Resultados}

Para Ryff (1989) el Bienestar Psicológico varía según la edad, el género, la cultura, los factores económicos, la desigualdad social, las diferencias políticas, la pobreza, entre otros; aspectos que en su conjunto van a orientar la significación que cada individuo atribuye a sus vivencias. Esto se relaciona con el análisis de las entrevistas realizadas a los participantes, quienes en sus discursos presentaron tanto similitudes como divergencias, de allí que serán expuestas en dos subapartados la agrupación de las cinco dimensiones sugeridas por Casullo (2002) y que coinciden con las categorías previas del estudio: Aceptación de sí mismo, Autonomía y Control de situaciones; Vínculos psicosociales y Proyectos. De cada dimensión, se destacarán también sus respectivas categorías emergentes.

\section{Aceptación de sí mismo, autonomía y control de situaciones}

La primera categoría emergente que aparece en relación con la dimensión Aceptación de sí mismo son las fortalezas que cada uno de ellos perciben en sus vidas. Se aprecia que pueden ser de dos tipos: fortalezas en lo interno, referidas a aspectos propios del individuo, como las cualidades, sentimientos, actitudes o características estructurales de cada uno. Y las fortalezas en lo externo, es decir, aquello que los individuos mismos relatan como elementos exteriores que han aportado a su estabilidad actual, tal como así lo enuncia una de las participantes: "mi fortaleza Dios, después de Él, mis tres Luceros: padre, madre e hija" (E. 3).

Sin embargo, la Aceptación de sí mismo no se refiere únicamente al reconocimiento de aquello positivo, también se enlaza con la capacidad que tiene la persona de identificar los componentes negativos pertenecientes al pasado o al presente, para posteriormente pensar en aquello que deben mejorar; por ejemplo, uno de ellos alude a su posición frente a la soledad:

"es duro cuando uno empieza a apropiarse de la soledad y de los motivos de la soledad, la verdad es que yo empecé a apropiarme ya estando en cambio, pues ya cuando empecé a cambiar" (E.1)

En cuanto a la dimensión Autonomía, ella se refleja en dos extremos, una asociada a la búsqueda de aprobación, y otra, en un afán por imponer sus deseos y opiniones sin importar lo que los demás piensen. En las siguientes dos citas se ilustra esta polaridad: 
"si querés que te diga la verdad, soy muy inseguro, y hablo mucho con el doctor...Pero primero me tiene que dar una opinión pa yo reconocer que soy capaz" (E.2)

"he aprendido en esta vida mi amor, que no es lo que tú me digas y mucho menos tú, ni lo que me diga el resto del mundo porque soy yo como tal, vuelvo y les repito, la que formo, pinto y sigo mi realidad solo mi realidad, mi presente como tal, si en este momento a mí se me pasa algo por la cabeza, que aguante" (E4)

Por su parte, en la categoría Control de situaciones, asumir la responsabilidad de los actos alude a un reconocimiento de que la vida actual depende únicamente de sus decisiones pasadas, al igual que su vida de calle en su momento fue consecuencia de una decisión propia. Lograr responsabilizarse de esas decisiones y no buscar culpables externos fue un aspecto fundamental en su resocialización, pues reconocieron que tienen la capacidad de tomar las riendas de sus propias vidas y que pueden adaptarse a las nuevas situaciones que se les presenten, sin que estas los desborden y los obliguen a recaer en el consumo y, como consecuencia de ello, volver a la calle.

Por otro lado, si bien en líneas anteriores se expone un desarrollo en la capacidad de modificar situaciones en los individuos abordados, no puede desconocerse que algunos de ellos expresaron sentir aún ciertos sentimientos displacenteros como la rabia o la frustración al momento de enfrentar un problema o una situación inesperada; sin embargo, estos sentimientos no impiden que los sujetos hallen la manera de direccionar o de transformar estas situaciones inesperadas o problemáticas para lograr satisfacer sus necesidades o deseos. Ante la presencia de estas situaciones generadoras de sentimientos de displacer, suelen utilizar la fe o confianza en Dios, pues expresan que orando logran calmar esos sentimientos y es lo que les da la fortaleza para afrontar cualquier tipo de situación.

\section{Vínculos psicosociales y proyectos}

Se aprecia una orientación hacia el bienestar en personas que han tenido un grupo familiar con quienes entablan vínculos positivos, de empatía y confianza, informaciones que aparecen con énfasis en la categoría Vínculos psicosociales, evidenciado en fragmentos como los siguientes:

"Entonces, el hecho de yo haber recuperado a mi hermana, de que mi hermana se enfrentaba a esas presiones de ella... El hecho de que mi hermana 
se enfrentó en cierta forma a la familia nuclear de ella, al esposo, a los hijos... Yo decía: Bueno, yo no puedo defraudarla a ella" (E.1)

"Mi familia me dijo cuando se canse, esta es su casa nunca me dieron la espalda, tengo muy buenos hermanos, muy buena familia...cuando se canse esta es su casa deje su vida un vínculo positivo" (E.3)

Finalmente, se puede plantear que, en relación con las demás categorías expuestas, la categoría Proyectos mostró una diversidad de factores tanto intrapersonales como extrapersonales que influyen en la manera en que cada persona otorga un sentido a su existencia y es precisamente ese sentido el que encamina sus metas y propósitos. De este modo, aunque las historias de vida de los entrevistados sean similares en numerosos aspectos, sus aspiraciones, maneras de alcanzar lo que se proponen y tiempos para lograrlo, son muy diversos; sin embargo, pese a esas diferencias, se puede apreciar que todos ellos han logrado encontrar un propósito y un sentido de vida.

En referencia a las metas de las personas abordadas, se encuentra que, como es normal en cualquier persona, tienen un orden cronológico, con la salvedad de que en estas personas su verdadero proyecto de vida comienza al salir de las calles, desde allí sus objetivos fueron variando, dependiendo del momento en el que se encontraran en su proceso de resocialización y de su ciclo vital. De este modo, dichos objetivos en primera instancia se fundamentaban en cubrir unas necesidades básicas que en su vida de calle no tenían, posteriormente, sus planes apuntaron más hacia recuperar sus vínculos familiares $\mathrm{y}$ sociales y en el momento actual se encuentra que sus metas giran en torno a lograr su autorrealización.

También consideraron necesario superar la farmacodependencia, asunto que según los relatos escuchados fue bastante complejo para todos, dado que como ellos mismos lo expresan, las recaídas son frecuentes. Aunado a ello, los participantes relatan haber vivido condiciones de salud muy delicadas que los llevaron a estar al borde de la muerte, por lo cual otro objetivo fundamental para ellos fue adoptar hábitos de vida más saludables y ser responsables en cuanto a los procedimientos médicos que debían realizarse para lograr un estado óptimo de salud en la medida de lo posible. Una vez superado este objetivo, apareció una nueva meta que era conseguir un empleo con el cual cubrir necesidades básicas, como vivienda, vestido y alimentación, y una vez alcanzada, se constituyó en un primer paso para la aceptación propia, ya que pudieron sentirse personas útiles y productivas.

Otro de los objetivos fundamentales para estas personas, fue la reconstrucción de sus vínculos familiares y sociales, todos ellos expresan que fue preciso 
alcanzar primero, al menos hasta cierta parte, los objetivos ya nombrados, para por medio de ello poder demostrarles a sus seres queridos su deseo real de rehabilitarse y de empezar una nueva vida e ir al mismo tiempo ganando poco a poco esa confianza perdida por todos los actos que llegaron a cometer debido particularmente a su adicción a las sustancias psicoactivas y permanencia en calle.

Estas son metas ya alcanzadas y hacen parte del pasado de las personas entrevistadas, pero son mencionadas por tres aspectos fundamentales: el primero de ellos es exponer la importancia que tiene para ellos destacar una a una las cosas que han ido logrando y que, por pequeñas o comunes que parezcan, han sido grandes retos; el segundo aspecto radica en resaltar cómo para estas personas su verdadero proyecto de vida comienza justo en el momento en que toman la decisión de abandonar las calles; y en tercer lugar, se muestra nuevamente la conexión que todas las categorías de Bienestar Psicológico tienen entre sí y cómo, para el caso puntual de la dimensión Proyectos, es esencial lograr un fortalecimiento en las demás dimensiones para encontrar un sentido de vida.

Ante este asunto, dos de los entrevistados manifiestan aún sentir temor de recaer en el consumo de sustancias psicoactivas, uno de ellos afirma que tener objetivos a largo plazo implica comenzar a ahorrar y que poseer una amplia suma de dinero en su poder puede ser una tentación para consumir; el otro participante refiriéndose a la importancia del trabajo en su vida, afirma que aunque ha tenido cantidades considerables de dinero en sus manos, no ha sentido la necesidad de la droga, sin embargo, prefiere evitar esto y vivir el día a día comprando de a poco lo que necesita para subsistir, porque el riesgo siempre está presente. Es importante resaltar que las personas en mención llevan tres y doce años fuera de las calles respectivamente, lo cual indica que no necesariamente a menor tiempo de haber dejado las calles es mayor el miedo a tener una recaída, pues el temor sigue presente con el pasar de los años y por eso estas personas buscan tener una ocupación o un propósito que los mantenga alejados de ello.

Aparece un aspecto que en particular llama la atención, dado que es expresado por todas las personas participantes del estudio y es el hecho de que de alguna forma todos ellos han sabido salir adelante y convertir todas las situaciones difíciles en aprendizajes, a partir de los cuales surge en ellos como propósito de vida servir a otros. Ellos expresan que por sus vivencias tienen la capacidad de comprender de una manera más integral a quienes están habitando la calle y por ello cada uno se ha propuesto realizar una especie de trabajo social desde diferentes ámbitos, por ejemplo, unos se desempeñan como 
educadores en centros de resocialización, otros son voluntarios que trabajan en pro de la rehabilitación de estas personas y otros simplemente expresan su plena disposición en participar en estudios como el presente, ya que afirman que sus historias de vida son un aporte valioso para comprender esta problemática. De este modo, puede evidenciarse cómo ayudar a quienes viven la misma situación que ellos vivieron en el pasado, es una razón que los motiva a seguir adelante y se convierte en uno más de los elementos que da sentido a sus vidas, pues, como ellos mismos lo expresan, "si Dios los tiene aún en este mundo a pesar de sus equivocaciones, es porque tienen una misión por cumplir", la cual es interpretada por ellos como servir al prójimo.

\section{Discusión}

Aunque en el presente estas personas tienen una visión positiva de la vida y una manera constructiva de enfrentar la adversidad, no desconocen que en ellos hay aún aspectos percibidos como negativos, por ejemplo, la forma de hablar, la inmediatez, la dependencia, la búsqueda de aprobación y la inseguridad en relación a la apariencia física, evidenciándose a partir de ello, un reconocimiento de atributos, que si bien no son positivos, son identificados como asuntos que pueden ser mejorados; esto, por consiguiente, promueve la aceptación de sí mismo en su integralidad (tanto lo positivo, como lo negativo). Dichos hallazgos también se han encontrado en otros estudios, como el de Casullo (2002), quien menciona en su investigación con adolescentes, que la dimensión Aceptación de sí mismo está descrita desde la forma de ser, las equivocaciones y la intención de mejorar, al mismo tiempo que se refiere a aceptar la vida que les tocó vivir y sus defectos.

Por otro lado, Mayordomo, Sales, Satorres y Meléndez (2016) en su estudio sobre Bienestar psicológico en función de la etapa de vida, el sexo y su interacción, concluyen que la dimensión Autonomía aumenta conforme aumenta la edad, coincidiendo con los trabajos de Ryff (1989), Ryff y Keyes (1995) y Casullo (2002), quienes indican que esta dimensión sigue un patrón incremental con la edad, desde la juventud hasta la ancianidad. Si bien estos estudios referenciados son de tipo cuantitativo, en el presente trabajo puede corroborarse nuevamente lo expuesto por los autores citados, dado que, en los relatos de los participantes, en su etapa de juventud contaban con un nivel muy bajo de autonomía y las distintas dinámicas familiares que rodeaban a cada uno de los individuos entrevistados contribuyeron a ello. En este orden de ideas, Mayordomo et al. (2016) concluyen que no hay diferencias significativas entre adultos y adultos mayores, lo cual sugiere que existe cierta estabilidad en la autonomía una vez alcanzada la adultez. 
En Casullo (2002), también es posible evidenciar la importancia que se le da al contexto cercano al indicar que a medida que la autopercepción de Bienestar disminuye, las áreas vitales -familia, amigos, otras relaciones interpersonales- se verían afectadas. Otros autores a favor de lo expuesto, indican que el apoyo, específicamente el familiar, es el principal predictor de la satisfacción vital y uno de los elementos más relacionados con el Bienestar Psicológico, en cuanto representa para la persona la sensación de estar acompañado y ser parte de una comunidad próxima que conforma una red social de ayuda, aporta en la realización de metas (Díaz y Sánchez, 2001) y se relaciona con la protección de la salud mental (Vivaldi y Barra, 2012). Asimismo, Casullo (2002), Díaz y Sánchez (2001) y Palomar y Cienfuegos (2007) encuentran que la fuente más importante de apoyo social y la motivación están representadas en los vínculos de confianza; no obstante, esta característica se ve contrarrestada por la confianza y autonomía que la persona resocializada tiene en sí mismo y de la consolidación de las formas en las que busca mantenerse en la situación actual.

Por otra parte, Vivaldi y Barra (2012) indican que una diferenciación entre aquello que es significativo en términos de calidad y no de cantidad tiene efectos en la selección de aquel que representa un apoyo social. De allí que, por ejemplo, Mayordomo et al. (2016), hayan identificado que a medida que se va entrando a la adultez, la cantidad de nuevos contactos sociales disminuye, y por eso los vínculos se reducen a las relaciones más estrechas con la familia y la pareja "teniendo en cuenta la calidad de esas relaciones y no la cantidad de las mismas" (p.109). Sin embargo, esa selectividad puede resultar también siendo un factor de riesgo que afecte el Bienestar Psicológico a nivel de la dimensión Vínculos psicosociales en personas resocializadas, pues este tipo de población caracterizada por tener unas historias de sufrimiento y desprendimiento de sus vínculos básicos representados por la familia y pareja, tuvo que sortearse en situaciones de maltrato o robo, y por eso para su supervivencia debió aumentar su suspicacia frente al otro como medida de protección. En este sentido, la selectividad para el establecimiento de relaciones en esta población es aún más exigente respecto a la calidad.

Frankl (1994) entiende el ser humano como un ser que ante la realidad elige y busca sustentarse en las personas que lo rodean y a quienes, a su vez, también intenta ayudar, de esta manera, reconoce la importancia de la libertad con la que cuenta el ser humano para elegir y la responsabilidad con que toma y asume sus decisiones; con base en esto, se comprende que en las personas entrevistadas el hecho de ayudar a otros se convierte en la razón que da sentido a sus vidas, pues es una elección y una decisión de vida propia. 


\section{Conclusiones}

Se puede afirmar que, si bien es muy importante trabajar en el fortalecimiento de todas las dimensiones que componen el Bienestar Psicológico en las personas que toman la decisión de dejar su vida de calle y rehabilitarse, si no se realiza un trabajo arduo por fortalecer la dimensión Proyectos, los esfuerzos realizados en las demás dimensiones serán en vano, pues son las metas y propósitos las que otorgan una motivación para vivir.

El principal aporte de la presente investigación está dirigido a los profesionales cuyo trabajo se centra en poblaciones vulnerables y más específicamente en la población habitante de calle. De este modo, esta perspectiva de comprensión del fenómeno en cuestión posibilita que el trabajo se fundamente en el fortalecimiento y potencialización de las dimensiones que componen el BP, con el fin de lograr una resocialización que permanezca en el tiempo, reconociendo la importancia del seguimiento tanto del individuo como de su red de apoyo familiar y social. Cabe destacar que esto se puede implementar no sólo cuando la problemática como tal ya se ha presentado, es decir, cuando la persona ya ha caído en las calles y lo que se busca es contrarrestar los perjuicios ya padecidos, sino también en actividades de promoción de la salud mental y la prevención de los factores de riesgo que predisponen a la vida de calle. Finalmente, permite dirigir la mirada hacia otros aspectos como el manejo del dinero, el autocuidado de la salud y la relación con las drogas que influyen en la consolidación del bienestar psicológico y que deben tenerse en cuenta si se quiere trabajar en torno a dicho concepto.

\section{Referencias}

Agudelo, A., Sánchez, M. (2012). Motivaciones de los y las habitantes de calle para ingresar a Centro Día en el componente de resocialización (Trabajo de pregrado de Psicología). Universidad de Antioquia, Medellín, Colombia.

Arias, A., Pamplona, J. (2015). Razones de hombres jóvenes habitantes de calle, entre los 20 y 50 años, para continuar viviendo en la calle (Trabajo de pregrado de Psicología). Universidad de Antioquia, Medellín, Colombia.

Barreat, Y. (2007). Estudio psicosocial de la indigencia en Mérida. Revista de Facultad de Medicina, Universidad de los Andes, 16 (1): 13-23. Disponible en http://erevistas. saber.ula.ve/index.php/medula/article/view/5743

Calderón, G., Gómez, M., Dávila, L., Zapata, J. (2018). Factores de logro en procesos de resocialización del habitante en situación de calle y consumidor de sustancias 
psicoactivas en Medellín, Colombia. Salud y drogas, 18 (2): 143-154, DOI: 10.21134/haaj.v18i2.387

Castro Solano, A., Casullo, M. (2001). Rasgos de personalidad, bienestar psicológico y rendimiento académico en adolescentes argentinos. Interdisciplinaria, 18 (1): 65-85, Disponible en https://www.redalyc.org/pdf/180/18011326003.pdf

Casullo, M., Castro Solano, A. (2000). Evaluación del bienestar psicológico en estudiantes adolescentes argentinos. Revista de psicología de la PUCP, 18 (1): 35-68, Disponible en http://revistas.pucp.edu.pe/index.php/psicologia/article/ view/6840

Casullo, M. M. (2002). Evaluación del Bienestar Psicológico en Iberoamérica. Buenos Aires: Paidós.

Correa, M. (2007). La otra ciudad - Otros sujetos: los habitantes de la calle. Revista del Departamento de Trabajo Social de Ciencias Humanas, Universidad Nacional de Colombia, 9: 37-56. Disponible en https://revistas.unal.edu.co/index.php/ tsocial/article/view/8511/9155

Departamento Administrativo Nacional de Estadística (DANE) y Alcaldía Metropolitana de Medellín (2002). Informe final del censo sectorial de habitante de y en la calle. DANE, Medellín.

Díaz, J.F., Sánchez, M. P. (2001). Relaciones entre estilos de personalidad y satisfacción autopercibida en diferentes áreas vitales en estudiantes universitarios. Psichotema, 14 (1): 100-105, Disponible en http://www.psicothema.com/pdf/692.pdf

Díaz, D.; Rodríguez, R.; Blanco, A.; Moreno, B.; Gallardo, I.; Valle, C., Dierendonck, D. (2006). Adaptación española de las escalas de bienestar psicológico de Ryff. Psicothema, 18 (3): 572-577, Disponible en http://www.psicothema.com/ psicothema.asp?id $=3255$

Di-Lorio, J., Seidman S., Gueglio, C., Rigueiral, G. (2016). Intervenciones psicosociales con personas en situación de calle: El cuidado como categoría de análisis. Psicoperspectivas. 15(3), 123-134, Disponible en http://dx.doi.org/10.5027/ psicoperspectivas-Vol15-Issue3-fulltext-838

Frankl, V. (1994). La voluntad de sentido. Barcelona: Herder S.A.

Galeano, M., Vélez, O. (2002). Estado del arte sobre fuentes documentales en investigación cualitativa. Medellín: Universidad de Antioquia. Centro de Investigaciones Sociales y Humanas.

González, M., Blandón, D., Quiceno, J., Giraldo, Á., Forero, C. (2014). Habitar bajo los puentes: vida y muerte; dos formas de comenzar algo. Revista Nacional de 
Salud Pública 32 (2): 36-41, Disponible en http://www.scielo.org.co/pdf/rfnsp/ v32n2/v32n2a05.pdf

Mayordomo, T., Sales, A., Satorres, E., Meléndez, J. (2016). Bienestar psicológico en función de la etapa de vida, el sexo y su interacción. Pensamiento Psicológico, 14(2): 101-112, Disponible en http://dx.doi.org/10.11144/Javerianacali.PPSI142.bpfe

Palomar, J., Cienfuegos, Y. (2007). Pobreza y Apoyo Social: Un Estudio Comparativo en Tres Niveles Socioeconómicos. Revista Interamericana de Psicología, 41 (02): 177-188, Disponible en: http://www.redalyc.org/articulo.oa?id=28441208

Restrepo Escobar, S., Storti Calderón, M. (2011). Propuesta metodológica. Articulación del programa de atención en farmacodependencia desde todos los componentes del sistema de atención al habitante de calle adulto. Modelo de reducción del daño y minimización del riesgo. Modalidad ambulatoria abierto y semiabierto. Medellín: Alcaldía de Medellín, Secretaría de Bienestar Social.

Ryff, C. D. (1989). Happiness is everything, or is it? Explorations on the meaning of psychological well-being. Journal of Personality and Social Psychology, 57, 1069-1081, Disponible en https://pdfs.semanticscholar. org/7eb5/1dfece4f39df7c5c3aefa1276ae1116473a5.pdf

Ryff, C. D., Keyes, C. (1995). Scale of Psychological Well-being. The structure of Psychological well- being revisited. Journal of personality and social psychology. 69, 719-727, Disponible en http://midus.wisc.edu/findings/pdfs/830.pdf

Secretaría de Bienestar Social y Centro de Estudios de Opinión de la Universidad de Antioquia. (2009). Realización del Censo de habitantes de calle y en calle de la ciudad de Medellín y sus Corregimientos. Revista electrónica la sociología en sus escenarios, 21. Disponible en https://aprendeenlinea.udea.edu.co/revistas/index. php/ceo/article/view/7073/6486

Vielma, J., Alonso, L. (2010). El estudio del bienestar psicológico subjetivo. Una breve revisión teórica. Educere, 14 (49), 265-275. Disponible en http://www.redalyc. org/articulo.oa?id $=35617102003$

Vivaldi, F., Barra, E. (2012). Bienestar Psicológico, Apoyo Social Percibido y Percepción de Salud en Adultos Mayores. Terapia psicológica, 30 (2), 23-29. Disponible en https://scielo.conicyt.cl/scielo.php?pid=S0718$48082012000200002 \&$ script $=$ sci_arttext\&tlng $=\mathrm{pt}$ 